\title{
La asociatividad y las cadenas productivas: una alternativa de desarrollo para el municipio de Yopal, Casanare
}

\author{
Luz Stella Cáceres Gómez ${ }^{\star}$, Claudia Esmeralda Pardo Enciso ${ }^{\star \star}$, Alicia Milena Torres ${ }^{\star \star \star}$
}

\begin{abstract}
Resumen
El presente trabajo realizado por el Grupo Lasallista de Economía Solidaria (GLEs) de la Universidad de La Salle, en desarrollo del proyecto titulado "Organización alternativa para la producción agrícola y turística en Yopal, Casanare", articulado al programa "Bioasentamientos humanos para una nueva ruralidad", que contribuye a la organización de pequeños y medianos productores agrícolas y a la dinamización de la actividad turística por medio de formas asociativas en las organización de cadenas productivas agrícolas en Yopal, Casanare, desde un enfoque de nueva ruralidad, con perspectiva hacia el 2014. La estructura del análisis observa la actividad productiva en Yopal y la conformación de cadenas productivas con fundamento asociativo como estrategia viable, considerando las ventajas comparativas presentadas en la región, el impacto de la actividad petrolera en la dinámica productiva, social, cultural y económica del municipio, y el marco de política nacional y regional que evidencia posturas gubernamentales hacia el fomento del desarrollo económico y social tanto del país como de las regiones, soportado en la dinamización de la actividad agrícola y turística.
\end{abstract}

Palabras clave: asociatividad, cadena productiva, economía solidaria, nueva ruralidad.

\section{Partnerships and Production Chains: A Development Alternative for the Municipality of Yopal, Casanare}

\begin{abstract}
This study was conducted by Grupo Lasallista de Economía Solidaria (GLES) of Universidad de La Salle, in the project titled "Alternative organization for agricultural and tourism production in Yopal, Casanare". This is linked with the "Human biosettlement for a new rural region" program which contributes to the organization of small and medium agricultural producers and the creation of dynamic growth in tourism through associative forms in agricultural production chain organizations in Yopal, Casanare. This work is conducted with a focus on new rural areas, looking towards 2014. The analysis examines production in Yopal, and the configuration of production chains with an associative basis and viable strategy, taking into account the region's relative advantages, the impact of oil production in the production, social, cultural and economic dynamic of the municipality, as well as the national and regional political framework that demonstrate the government's approach to promoting economic and social development of the country and the regions supported by dynamic agriculture and tourism.
\end{abstract}

Keywords: associativity, production chain, solidarity-based economy, new rural areas.

\section{A associatividade e as cadeias produtivas: uma alternativa de desenvolvimento para o município de Yopal, Casanare}

\section{Resumo}

O presente trabalho realizado pelo Grupo Lasallista de Economia Solidária (GLES) da Universidade de La Salle, em desenvolvimento do projeto intitulado "Organização alternativa para a produção agrícola e turística em Yopal, Casanare" (Colômbia), vinculado ao programa "Bioassentamentos humanos para uma nova ruralidade", que contribui para a organização de pequenos e médios produtores agrícolas e para a dinamização da atividade turística por meio de formas associativas na organização de cadeias produtivas agrícolas em Yopal a partir de um enfoque de nova ruralidade, com perspectiva para 2014. A estrutura da análise observa a atividade produtiva em Yopal e a conformação de cadeias produtivas com fundamento associativo como estratégia viável, que considera as vantagens comparativas apresentadas na região, o impacto da atividade petroleira na dinâmica produtiva, social, cultural e econômica do município, e o marco de política nacional e regional que evidencia posturas governamentais ao fomento do desenvolvimento econômico e social tanto do país quanto das regiões, suportado na dinamização da atividade agrícola e turística.

Palavras-chave: associatividade, cadeia produtiva, economia solidária, nova ruralidade. 


\section{Introducción}

El trabajo que se presenta a continuación da cuenta de la primera fase de la investigación que adelanta el grupo GLES, orientada a identificar las condiciones existentes que permitan la organización y el desarrollo de cadenas productivas agrícolas y de turismo en el municipio de Yopal, soportado en formas asociativas de organización para la producción.

Este primer acercamiento se fundamenta en el análisis del comportamiento de la actividad agrícola y turística de Casanare, específicamente del municipio de Yopal, mediante el estudio del desempeño de tres factores: desarrollo institucional, cadenas productivas y asociatividad, como germen de los procesos de economía solidaria que cimientan una visión del desarrollo, de acuerdo con una propuesta de "nueva ruralidad". Dichos aspectos definen las condiciones con que cuenta la región, para incluir a los pequeños productores en los procesos de desarrollo rural, soportados en la promoción de la soberanía alimentaria como estrategia de autonomía económica y política que dinamiza su vocación agrícola.

El desarrollo de esta primera fase proporciona información necesaria sobre los elementos que constituyen la base de la estructura y el desarrollo de las fases II y III de la investigación, la cual culminará con una propuesta de fortalecimiento de la organización de los pequeños productores a través de las alianzas que se establezcan con el sector público y privado, como soporte imprescindible para la materialización de este emprendimiento solidario.

Inicialmente se presentan los fundamentos teóricos y conceptuales que dan soporte a la investigación y que guían el desarrollo de la propuesta; a continuación se contextualiza la actividad agrícola en el panorama socioeconómico de la región, con el fin de establecer su importancia en relación con las otras actividades económicas locales; posteriormente se plantea un análisis de documentos de política pública plasmados en el Plan de Desarrollo, en las agendas de competitividad, en los documentos Conpes, en las alianzas estratégicas regionales, entre otros. Finalmente, se analiza el avance que ha tenido la organización del sector agrícola y el desarrollo de las cadenas productivas, lo mismo que el papel que ha jugado la asociatividad en este proceso.

\section{Revisión conceptual}

La globalización, como fenómeno mundial que permea la vida de las personas, la manera como se relacionan, la dinámica económica, las demandas de bienes y servicios, las innovaciones tecnológicas, además de la regulación de la producción y del consumo, trajo consigo un paradigma de integración mundial basado en la homogeneización económica cultural y política, a través del modelo neoliberal, soportado en la individualización de la sociedad y en el urbanismo, como ejes del cambio que convoca a salir del "atraso y la ignorancia", calificativos con los que se señala a las poblaciones rurales. Esta dicotomía campo-ciudad proporciona la construcción de imaginarios sociales que desestimulan las actividades del campo y acarrea una gran concentración de población en las grandes ciudades en busca de nuevos horizontes que les permita integrarse a las dinámicas económicas urbanas para acceder a los beneficios que el "desarrollo" de la ciudad suministra a sus habitantes.

El concepto de "nueva ruralidad" propone una visión integradora que conjuga las actividades del campo con las de la ciudad, como formas complementarias e imprescindibles para la existencia de la vida, al concebir la "región" como unidad sistémica que rompe con la polaridad y permite nuevas construcciones sociales, económicas y políticas que le dan visibilidad desde las particularidades que la caracterizan para ser reconocida dentro de la diversidad, mediante políticas públicas y estrategias especiales que pueden dar respuesta a sus propias necesidades.

La "nueva ruralidad" señala la necesidad de superar las concepciones de lo rural como lo agrario, para asignarle un carácter multifuncional, desde el que se reconozca la gran diversidad de actividades que pueden desarrollarse en el campo, además de las agrícolas, y empieza a mostrar una visión del territorio ${ }^{1}$ como eje

1. Como enfoque teórico importante la "nueva ruralidad" integra lo rural con lo urbano de acuerdo con el concepto de territorio, con el fin de romper esta dicotomía que conlleva a concebir la ciudad como espacio de desarrollo y al campo como sinónimo de atraso y pobreza. Según Ávila (1999), territorio es la "construcción que opera gracias a las interacciones entre actores y actividades pero sobre todo por la puesta en práctica de estrategias operacionales que se apoyan sobre la cultura, la historia, las movilizaciones del "saber hacer", los fenómenos de aprendizaje colectivo, los modos o formas de cooperación y de asociación en tre los actores y las prácticas de adaptación que se desarrollan en escala local”. (p. 223). 
del análisis y no la dispersión demográfica de la población como casi único factor de categorización de los espacios rurales" (Pérez y Farah, 2006, p. 86).

Así como la "nueva ruralidad" propone otras posibilidades económicas generadoras de ingresos más allá de la actividad agrícola - como por ejemplo el turismo, el cual debe desarrollarse con fundamento en la apropiación cultural de la riqueza del paisaje - también plantea la importancia de fortalecer la participación ciudadana en los espacios de toma de decisiones. Esto implica que los productores actúen como actores sociales que velan por la defensa de sus derechos, no sólo como agentes productivos sino como sujetos sociales y políticos con derechos y responsabilidades individuales y colectivas.

Asimismo, se considera la solidaridad como principio y valor que da sentido y capacidad para comprender y actuar sobre la realidad, que orienta la consecución de beneficios sociales para el colectivo, otorgando preponderancia a las interacciones por medio de la cooperación. Este enfoque hace manifiesto un compromiso con el entorno social y ambiental, propicia la generación de pequeñas organizaciones y estimula su organización coordinada dentro de contextos económicos plurales.

Es así como para Singer, citado por Ghibaudi (s. f.), lo que mejor caracteriza y fundamenta el modelo de economía solidaria es la cooperación entre individuos que deciden construir emprendimientos socioeconómicos - y también sus redes- mediante una propiedad y gestión conjunta e igualitaria, estableciendo así relaciones basadas en la solidaridad y no en la competencia. Así, esto es lo que determinaría - y más claramente explicaría- que puedan desarrollar una lógica de relaciones sociales orientadas a la reproducción y realización de sus participantes.

El surgimiento de la economía solidaria con el auge del cooperativismo en el siglo XVIII, como alternativa al modo de producción capitalista, ha demostrado ofrecer grandes beneficios para quienes piensan que la unión con otros, desde figuras como las asociaciones, empresas colectivas, fondos y otras formas de organización, facilita la solución de las problemáticas económicas y sociales que plantea la sociedad moderna.

Otro actor importante en esta propuesta de desarrollo rural es sin duda el Estado, el cual interviene por medio de la política pública entendida, según Vargas (1999), como el conjunto de sucesivas iniciativas, decisiones y acciones del régimen político frente a situaciones socialmente problemáticas y que buscan la resolución de estas o llevarlas a niveles manejables.
El mismo autor señala que en el diseño y la implementación de la política pública juegan un papel importante todos los actores locales que hacen presencia social y cuentan con el poder suficiente para incidir en la definición y el desarrollo de ellas. Toda política pública permite que unos sectores se sientan más favorecidos que otros, por lo tanto cada sector buscará presionar a la administración para lograr ser tenidos en cuenta y así obtener beneficios en la definición y la implementación de dichas políticas. Ellas requieren ser explicadas y consensuadas para convencer a la opinión y reducir la oposición, y por tanto su éxito depende del nivel de comunicación que logren para su difusión.

Otro factor de reflexión es el encadenamiento productivo que como forma asociada estratégica ha dado resultados exitosos en términos de cualificación de procesos, productos y servicios; de gestión de los actores participantes, tales como facilidad en flujos de información, disminución de costos, aumento en la capacidad de negociación y de generación de compromiso hacia la responsabilidad compartida por la calidad.

Las cadenas productivas se han considerado como una organización de agentes integrados o asociados en torno a la producción de un bien o de un servicio con potencial de mercado, que impulsan una dinámica y unas relaciones desde el momento de la producción, pasando por la transformación y la distribución hasta llegar a su consumidor o usuario final. También son definidas como "un conjunto articulado de actividades económicas integradas, consecuencia de articulaciones en términos de mercado, tecnología y capital" (Chevalier (2003), citado por Gómez, s. f., p. 4); así, se constituyen en estrategias para el desarrollo de ventajas competitivas de las empresas.

Para pequeños productores y comercializadores, estas oportunidades son alcanzables al lograr establecerse como grupo caracterizado por una confianza en la asociación como:

mecanismo de cooperación empresarial en el que cada una de las empresas que participan mantiene su independencia jurídica y su autonomía gerencial, decidiendo voluntariamente su participación en un esfuerzo conjunto con los otros participantes para la búsqueda de un objetivo común (Rosales, citado por Narváez et al., 2008).

De lo contrario, su esfuerzo de producir y llevar el producto al consumidor final será costoso y de menor competitividad, desalentando la iniciativa empresarial y obligando a tomar otras alternativas que lo alejarán 
de la posibilidad de esa independencia y autonomía ya referida.

Dicha cadena, entendida como estrategia colectiva capaz de generar valor agregado al proceso de producción o comercialización, se estructura en "el marco de redes formales e informales, relaciones y determinados eslabonamientos productivos con proveedores, clientes y mercados, reflejando experiencias de endogenización del desarrollo" (Narváez et al., 2008), en las cuales se destacan aspectos como los factores sociales, históricos, institucionales y ambientales en la transformación socioeconómica del territorio, así como las condiciones de calidad de servicios e infraestructura básica de apoyo a la producción.

\section{Entorno socioeconómico de la región del Casanare}

Hablar de desarrollo con respecto al municipio de Yopal y al departamento de Casanare, obliga a partir de las condiciones existentes en las que, al igual que en una gran cantidad de regiones colombianas, prevalecen concepciones divisorias entre lo rural y lo urbano, conflictos sociopolíticos complejos, emergencia de nuevos asentamientos humanos que contribuyen a generar rupturas inesperadas en el mundo rural de la Orinoquía, generación de cinturones de miseria con problemas de pobreza, indigencia y abandono de muchas de aquellas zonas denominadas rurales, con la correspondiente discriminación social, educativa, productiva, económica y de salud a la que son sometidos estos grupos poblacionales migrantes.

La región posee riquezas naturales, lugares exóticos que muestran diversidad de especies de flora y fauna, hermosas playas de ríos legendarios y diversas fuentes hídricas; historia, tradiciones y costumbres del llano casanareño y su gran riqueza de hidrocarburos que la hacen un centro acogedor e interesante para toda clase de visitante. Sin embargo, "la actividad turística en el municipio es muy incipiente y los pocos atractivos turísticos, se encuentran deteriorados y dan un mal aspecto especialmente para los turistas" (Yopal vive. 2010, p. 27).

Actualmente, la región de los Llanos Orientales, en cuyo territorio se encuentra localizado el departamento de Casanare, con capital en Yopal, vive una problemática socioeconómica derivada de la especialización en la actividad petrolera. "Para el 2010, el mayor crecimiento de la producción petrolera en el departamento con una variación del 52,3\% con respecto al 2009” (Municipio de Yopal, 2012, p. 13), cuyo impacto afecta la búsqueda de beneficios en las demás actividades productivas.

\section{Actividad petrolera en Yopal}

En la última década, el auge de la minería, y en especial de los hidrocarburos, ha determinado cambios significativos en la dinámica económica del país, tomando como base incrementos importantes en el rango de exportaciones del sector minero, de los cuales, en el 2002, representaban valores cercanos al 12,72\% del total del valor de las exportaciones nacionales, frente a las cifras del 2009, en las que representó el 24,82\% del total de exportaciones, es decir, presentó un incremento de 12,1 puntos porcentuales (Minminas, 2010). De acuerdo con esta situación, en el 2011 Yopal recibió en regalías 5.445 millones de pesos, siendo este el tercer municipio del país en recibir más dinero por este concepto, y en total el departamento del Casanare recibió 23.796 millones de pesos. Según lo establecido en la Ley 756 de 2002, estos dineros deberían ser invertidos en proyectos prioritarios de salud, educación y saneamiento básico, de acuerdo con el Plan de Desarrollo de las entidades territoriales beneficiarias (Gómez, s.f.).

El inicio de la actividad de extracción petrolera en los municipios de Casanare genera modificaciones en los imaginarios de la población sobre expectativas en la generación de empleo, producto de nuevas oportunidades laborales, ancladas a la actividad minera, en desmedro del aprovechamiento de la tierra, que implica un mayor esfuerzo inmediato, ganancias menores a largo plazo e incertidumbre en futuras inversiones propias de la actividad productiva. En razón a lo anterior, el incremento de la competencia entre los pobladores de las veredas por conseguir un trabajo en las petroleras, y la migración hacia la zona de población flotante, que incide directamente en la desencadenante transformación cultural y social, lo cual genera una dinámica de competencia por el logro de una opción laboral, así como un crecimiento demográfico no planificado y desbordado en la región y, aún más, un crecimiento geográfico desordenado por la búsqueda de territorio en la generación de nuevos asentamientos humanos para los cuales la localidad y los niveles de saneamiento básico no están preparados. 


\section{Metodología}

En primer lugar se procedió a realizar un análisis documental, visita de campo y entrevistas con líderes de los corregimientos con potencial productor, y reuniones con representantes de entidades públicas (alcaldía, gobernación, centros provinciales), responsables de formular y ejecutar políticas públicas orientadas al desarrollo de la producción agrícola y turística del municipio, así como a entidades privadas como la Fundación Amanecer, la Universidad del Trópico y la Universidad de San Gil.

Dado el objeto de estudio y las condiciones particulares del municipio de Yopal, se decidió optar por una metodología participativa que facilite la dinámica organizativa de los actores, como la metodología IAP (investigación-acción-participación), que le permite a los involucrados una resignificación de su realidad, al asumir el rol de investigadores, que les permite analizar su entorno, comprender y proponer formas viables para la articulación con su entorno.

\section{Resultados}

Desde lo regional, se puede señalar que los últimos Planes de Desarrollo del municipio de Yopal advierten que lo más significativo dentro de la problemática regional lo constituyen ciertas deficiencias de tipo estructural; esto obedece particularmente a circunstancias que se relacionan con un tejido social descompuesto, baja cobertura de servicios públicos, ausencia de factores de competitividad y productividad, y falta de integración territorial y de equidad.

De modo que el Concejo Municipal, en su Plan de Desarrollo "Yopal Vive el Cambio 2011”, prevé líneas estratégicas de acción encaminadas a "consolidar el proyecto ciudad-región, fortalecer el desarrollo productivo y económico, y establecer procesos de planeación territorial y de medio ambiente".

En este contexto, el eje programático que propende por un crecimiento productivo y sostenible, a nivel regional, define metas en torno a una economía de escala que permite fomentar sinergias y establecer alianzas basadas en la promoción de estrategias; en ese sentido, tal directriz implica considerar como posibles mecanismos de integración el trabajo colectivo, la asociatividad, el emprendimiento, la microempresa y la economía solidaria.

A su vez, se reconoce la necesidad de modernizar las actividades agrícolas y pecuarias de la región con miras a consolidar nuevos mercados en los espacios no sólo departamentales, sino también en otros escenarios nacionales e internacionales.

Los pequeños productores agrarios del departamento de Casanare, así como los de las demás regiones del país, están expuestos a la dispersión y falta de organización para el desarrollo de sus actividades productivas y de comercialización, lo cual genera un grave problema de sostenibilidad para mantenerse en el sector productivo y no son reconocidos como actores sociales en el complejo socioproductivo local. Si bien las autoridades gubernamentales, organizaciones públicas y privadas, universidades y líderes de corregimientos, expresan el interés y destacan la importancia de las formas asociativas como motores para el desarrollo productivo de pequeños y medianos empresarios, se presenta escasa confianza de los productores en los mismos agentes gubernamentales y entidades prestadoras de servicios de capacitación y consultoría, puesto que se han hecho intentos con muy poco impacto en el desarrollo del sector.

A propósito de la actividad agrícola empresarial del municipio de Yopal, actualmente se encuentran 6.600 hectáreas de arroz, con rendimientos promedio de 5 ton/ha, 920 ha en palma de aceite, 450 de plátano, 450 de yuca, 125 de caña miel, 83 de cacao, 22 de café, 22 de piña y 11 de maracuyá. La mayor área sembrada corresponde al arroz, constituida como la cadena con mayor tradición de la región, y que presenta ventajas comparativas nacionales, por la calidad del grano y los rendimientos por debajo de la media nacional (Plan de Desarrollo, 2010, p. 63), seguida por la palma de aceite, el plátano, la yuca, caña miel, cacao, café, piña y maracuyá.

Los nuevos escenarios económicos que plantea la puesta en marcha del tratado de libre comercio afecta la producción arrocera, haciendo indispensable una reflexión profunda sobre los virajes que debe dar la producción agrícola en este contexto. Así pues, surgen nuevas fórmulas productivas como la sustitución del cultivo de arroz por el de piña, maracuyá u otros cítricos como el limón y el cacao, ofreciendo incentivos económicos para los pequeños productores que se arriesguen a esta trasformación.

La relación existente entre el área sembrada y la producción de la piña (variedad golden) y del maracuyá, presentan niveles de rendimiento de 29 y 15 ton/ha, por lo que se generan oportunidades en términos de precios, agroindustrialización, iniciativa de productores por la producción a gran escala, demanda y calidad, 
los niveles de organización y de capacidad de liderazgo de sus productores, factor este último que aún presenta muchas debilidades.

Se encontraron condiciones de viabilidad para la yuca, por la existencia de planta procesadora de harina en cercanías a Yopal, antecedentes de producción e interés de productores potenciales de apostarle a grandes volúmenes de producción.

Líderes de corregimientos, autoridades gubernamentales, representantes de entidades de fomento privado y de universidades, coinciden en señalar la potencialidad productiva existente de los productos señalados, a partir de la organización en una cadena productiva y la necesaria alternativa de formas asociativas que faciliten la vinculación del pequeño productor. No obstante, también coincidieron en destacar la falta de confianza entre los productores, que será necesario trabajar al inicio de la segunda fase.

Sin embargo, una de las problemáticas principales en este contexto es la poca experiencia con que cuenta el municipio sobre el desarrollo de las cadenas productivas, toda vez que no se ha centrado en incluir a los pequeños productores sino, por el contrario, se han diseñado para los productos más importantes y que se han cultivado con visos de industrialización agrícola, como el caso del café y el arroz.

En cuanto a la cadena turística, se estableció la coincidencia por parte de los agentes entrevistados y del plan de desarrollo turístico (aún sin ejecutar), de que existen condiciones naturales que favorecen la actividad turística, así como otros factores como el patrimonio cultural, las festividades, los eventos grandes, diversidad de opciones para el acceso terrestre, la presencia de principales entidades financieras y cajeros automáticos, entre otros, y limitaciones como el deficiente estado de las vías y la señalización vial, la inexistencia de la señalización turística, la inadecuada infraestructura y el servicio del terminal de transporte terrestre, oferta hotelera y de restaurante escasa y con deficiente e inadecuada capacitación para empresarios y personal de servicios turísticos, entre otros.

\section{Conclusiones}

Los últimos dos planes de desarrollo del municipio de Yopal determinan la necesidad de apalancar el desarrollo económico y social del municipio en la dinamización del sector agrícola y turístico y, en consecuencia, han establecido, como motores del desarrollo, entre otros, la cadena frutícola y la cadena turística, iniciativas de política que fueron ratificadas por las autoridades municipales de Yopal, con miras a incluir la pequeña y mediana producción en este proceso.

La economía centrada en la explotación de hidrocarburos afecta la vida cultural y social de las comunidades productivas de Casanare, ya que minimiza la motivación de los pobladores hacia el agro, desencadenando un abandono de la actividad agrícola, resultante de la idea de obtener mejores ingresos económicos mediante la vinculación a las empresas petroleras, lo que determina comportamientos de espera sólo por aquellos trabajos mejor remunerados, ajenos a su actividad económica tradicional y un desborde por la cultura del consumismo que se observa en incrementos en los precios que afectan el costo de vida, generan inequidad en la distribución de la riqueza, nuevos referentes de modus vivendi y de estatus, inseguridad y prostitución. Unido a esto, la especulación en los precios de la tierra - ya que los pobladores ven como una oportunidad de negocio la venta de sus predios a las petroleras- incrementa aún más los impactos producto de este desarrollo.

De otra parte se encontró escasa credibilidad en el apoyo de agentes gubernamentales para el desarrollo y la continuidad de proyectos productivos, así como en la asistencia técnica permanente que garantice la productividad y competitividad esperada.

La desconfianza y la falta de cooperación entre los diferentes actores (públicos, privados y los pequeños productores) impide los procesos organizativos sostenibles, ya que la organización se da en torno a personas y no a procesos sólidos y fuertes que den visibilidad del sector socioproductivo.

Finalmente se establecieron acuerdos de trabajo interdisciplinario e interinstitucional con las organizaciones públicas, privadas, dirigentes de corregimiento y universidades, con el propósito de realizar conjuntamente el desarrollo de la segunda fase que está orientada a convocar a los potenciales productores y realizar las actividades previstas para la sensibilización de los actores hacia la organización asociativa, dejando sentadas las bases para realizar un trabajo mancomunado, con el propósito de impulsar dinámicas productivas y de turismo desde el enfoque de la economía solidaria como alternativa que permita la inclusión de los pequeños productores. 


\section{Referencias}

Avila. J. (1999), La ordenación del territorio en el país Vasco: Análisis ejercicio y delimitación competencial. Madrid: Civitas.

Gómez, D. (s. f.). Asociatividad empresarial y apropiación de la cadena productiva como factores que impulsan la competitividad de las micro, pequeñas y medianas empresas: tres estudios de caso. Recuperado de http:// www.pa.gob.mx/publica/rev_47/an\%C3\%A1lisis/asociatividad_empresarial.pdf

Minminas. (2010). Memorias 2010-03. Recuperado de http:// www.minminas.gov.co/minminas/downloads/UserFiles/File/Memorias/Memorias_2010/03-minas.pdf

Municipio de Yopal. (2008). Plan de Desarrollo del Municipio de Yopal para el periodo 2008-2011 "Yopal Vive el Cambio”. Recuperado de HTTP://YOPAL-CASANARE.GOV.Co/ APC-AA-FILES/38336239393262616235333063343464/ PLAN_DE_DESARROLLO_YOPAL_VIVE_EL_CAMBIO.pdf

Municipio de Yopal. (2012). Plan de Desarrollo "Yopal con sentido social 2012-2015". Recuperado de http:// www.yopal-casanare.gov.co/apc-aa-files/38373637613 335353231613733653633/plan_de_desarrollo_2012_ 2015_definitivo_com_medio_1.pdf

Narváez, M.; Fernández, G. y Senior, A. (2008). El desarrollo local sobre la base de la asociatividad empresarial: una propuestaestratégica.27,57.Opción:Maracaibo.Recupe-
radodehttp://www.scielo.org.ve/scielo.php?pid=S101215872008000300006\&script=sci_arttext

Pérez, E. y Farah, M. A. (2006). Nueva ruralidad en Colombia. Nueva ruralidad. Enfoques y propuestas para América Latina. México: Centro de Estudios para el Desarrollo Rural Sustentable y la Soberanía Alimentaria. Cámara de Diputados, Lx Legislatura.

Plan de desarrollo de Yopal, Casanare. Recuperado de http:// yopal-casanare.gov.co/apc-aa-files/346334396239 61383838323233376564/PLAN_DE_DESARROLLO_YOPAL_VIVE_EL_CAMBIO.pdf

Prensa Libre. Recuperado de http://www.prensalibrecasanare.com/principal/2131-casanare-recibiu-23796-millones-de-pesos-por-regalnas-en-octubre-yopal5445-millones.html

Singer, P. (2000), A economia solidária no Brasil. São Paulo: Contexto. Cibergrafía

Ghibaudi, J. (sf). Economía solidaria: su matriz conceptual y su objeto de estudio, recuperado el 10 de agosto de de 2012, de http://www.econ.uba.ar/www/institutos/ epistemologia/marco_archivos/Trabajos\%20Completos\%20IX\%20Jornadas\%20en\%20PDF/Ghibaudi\%20 Javier.pdf

Vargas, A. (1999). El Estado y las políticas públicas. Capitulo II. Las políticas públicas entre la racionalidad técnica y la racionalidad política. Bogotá: Almudena Editores. 